\title{
The impact of curved satellite tracks on SAR focusing
}

\author{
Mohr, Johan Jacob; Madsen, Søren Nørvang
}

Published in:

Proceedings of IEEE 2000 International Geoscience and Remote Sensing Symposium

Link to article, DOI:

10.1109/IGARSS.2000.860430

Publication date:

2000

Document Version

Publisher's PDF, also known as Version of record

Link back to DTU Orbit

Citation (APA):

Mohr, J. J., \& Madsen, S. N. (2000). The impact of curved satellite tracks on SAR focusing. In Proceedings of IEEE 2000 International Geoscience and Remote Sensing Symposium (Vol. 1, pp. 87-92). IEEE. https://doi.org/10.1109/IGARSS.2000.860430

\section{General rights}

Copyright and moral rights for the publications made accessible in the public portal are retained by the authors and/or other copyright owners and it is a condition of accessing publications that users recognise and abide by the legal requirements associated with these rights.

- Users may download and print one copy of any publication from the public portal for the purpose of private study or research.

- You may not further distribute the material or use it for any profit-making activity or commercial gain

- You may freely distribute the URL identifying the publication in the public portal 


\title{
The Impact of Curved Satellite Tracks on SAR Focusing
}

\author{
Johan Jacob Mohr and Søren Nørvang Madsen \\ Dept. of Electromagnetic Systems, Technical University of Denmark \\ Ørsteds Plads, Build. 348, DK-2800 Kgs. Lyngby, Denmark \\ Phone: +45 45253800 / Fax: +45 4593 1634 / E-mail: jm@emi.dtu.dk
}

\begin{abstract}
This paper addresses the geometric effect of processing single look complex synthetic aperture radar (SAR) data to a reference squint angle different from that given by the center of the real antenna beam. For data acquired on a straight flight line, the required transformation of radar coordinates from one Doppler reference to another is independent of the target elevation but for data acquired from a satellite orbit over a rotating Earth that is not true. Also the effect of ignoring Earth rotation is addressed.
\end{abstract}

\section{INTRODUCTION}

For large scale exploitation of satellite synthetic aperture radar (SAR) data geometric fidelity is often important. With the European Space Agency ERS-1/2 satellites it has been demonstrated that slant range images can be characterized geometrically with an accuracy corresponding to $10 \mathrm{~m}$ on ground by using a dead-reckoning approach, [1]. The final geocoding accuracy for conventional SAR brightness images depends on the accuracy of the digital elevation model used. For SAR products, geocoded using interferometric information, the accuracy depends on the scene and on the acquisition conditions (baseline, atmosphere, etc.) By paying attention to processing fidelity even current satellite SARs support a geometric accuracy on the order of $10 \mathrm{~m}$ without use of ground control points.

This paper addresses the impact of geometrically referencing SAR data to a zero Doppler geometry when the platform path is curved and the terrain elevation is unknown. The paper also addresses the large azimuth shift which occur if the horizontal curvature of the orbit due to Earth rotation is ignored.

\section{GEOLOCATION OF SAR IMAGES}

The fundamental equations characterizing the geometry of slant range SAR images are the range and Doppler equations

$$
\begin{aligned}
R & =\left|\bar{R}_{\mathrm{s}}-\bar{R}_{\mathrm{t}}\right| \\
f_{\mathrm{D}} & =-\frac{2}{\lambda R}\left(\bar{V}_{\mathrm{s}}-\bar{V}_{\mathrm{t}}\right) \cdot\left(\bar{R}_{\mathrm{s}}-\bar{R}_{\mathrm{t}}\right)
\end{aligned}
$$

where $R$ is the range, $f_{\mathrm{D}}$ the Doppler frequency, $\bar{R}$ a position vector, $\bar{V}$ a velocity vector, $\lambda$ the wavelength, and indices $\mathrm{s}$ and $\mathrm{t}$ denotes satellite and target, respectively, e.g. [2].

The analysis presented in this paper uses an Earth body fixed coordinate system, i.e. the target velocity is zero. Also, the
Doppler frequency, $f_{\mathrm{D}}$, is expressed as the squint angle, $\psi$, between the plane orthogonal to the velocity vector and the line-of-sight vector.

\section{LINEAR SENSOR PATH}

For a linear flight track slant-range images can be transformed between different viewing geometries without knowledge of target heights, [3]. An important special case is that a SAR image acquired on a straight flight track, independent of the squint at acquisition, can be transformed to a zero-Doppler geometry. The relevant transformations are

$$
\begin{aligned}
& r_{0}=r_{1} \cos \psi_{1}=r_{2} \cos \psi_{2} \\
& r_{0}=-s_{1} \cot \psi_{1}=-s_{2} \cot \psi_{2}
\end{aligned}
$$

where $r$ denotes slant range, $s$ the along track coordinate, and $r_{0}$ is the closest approach distance. The key to the equations is that the axis of the Doppler cone does not change with $s$.

The implication is that knowledge of the acquisition squint angle is not required for geocoding, only the reference squint angle, utilized in the processing (often zero) is required.

\section{SATELLITE ORBITS}

Near circular satellite orbits, $\bar{p}(s)$, have a curvature, $\kappa_{v}$, in the vertical plane of approximately $\kappa_{v} \approx 1 /\left(R_{e}+H\right)$, where $R_{e}$ denotes the Earth radius and $H$ the satellite height. The orbit also have a curvature, $\kappa_{h}$, in the horizontal plane due to Earth rotation. In polar regions $\kappa_{h} \approx 0.15 \kappa_{v}$ (for ERS and Radarsat) decreasing to zero near the equator.

We use a circular orbit approximation fitted to the orbit at a reference point, $\bar{p}\left(s_{0}\right)$, such that the unit tangent vectors of the reference and the actual tracks, $\hat{t}_{0}$, coincide, the unit vectors, $\hat{b}_{0}$ (out of orbit plane) and $\hat{n}_{0}$ (in-plane), coincide (i.e. identical osculation planes), and the curvatures, $\kappa\left(s_{0}\right)$, are identical. The unit vectors $\left(\hat{t}_{0}, \hat{n}_{0}, \hat{b}_{0}\right)$ are given by, [4],

$$
\begin{aligned}
& \hat{t}_{0}=\frac{\bar{p}^{\prime}\left(s_{0}\right)}{\left|\bar{p}^{\prime}\left(s_{0}\right)\right|} \\
& \hat{b}_{0}=\frac{\bar{p}^{\prime}\left(s_{0}\right) \times \bar{p}^{\prime \prime}\left(s_{0}\right)}{\left|\bar{p}^{\prime}\left(s_{0}\right) \times \bar{p}^{\prime \prime}\left(s_{0}\right)\right|} \\
& \hat{n}_{0}=\hat{b}_{0} \times \hat{t}_{0}
\end{aligned}
$$

With $R_{\kappa}=1 / \kappa\left(s_{0}\right)$, a natural representation of the orbit model, 


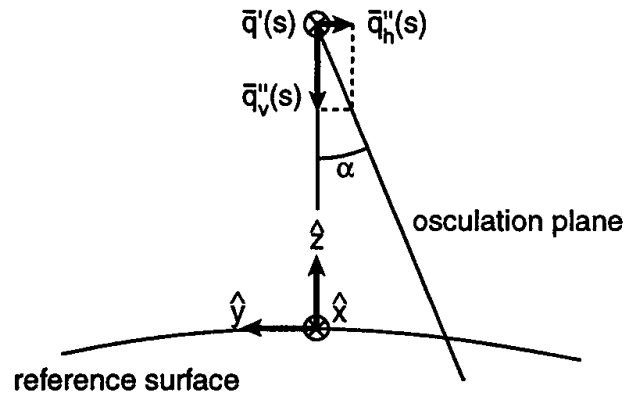

Fig. 1: Satellite orbit $\bar{q}(s)$ with an acceleration (curvature) in both the vertical direction, $\bar{q}_{v}^{\prime \prime}(s)$, and the horizontal direction, $\bar{q}_{h}^{\prime \prime}(s)$. The osculation plane of the orbit has an angle, $\alpha$, with the vertical.

$\bar{q}(s)$, can be written as

$$
\bar{q}(s)=R_{\kappa} \sin \frac{s}{R_{\kappa}} \hat{t}_{0}+R_{\kappa}\left(1-\cos \frac{s}{R_{\kappa}}\right) \hat{n}_{0}
$$

see Fig. 1. Note that the plane containing $\bar{q}(s)$ will in general not contain the nadir point. For ERS the osculation plane is rotated at most $9^{\circ} \approx \arctan 0.15$ relative to the vertical.

\section{CURVED SENSOR PATH}

For a curved flight track, slant-range images cannot be transformed to other viewing geometries without knowledge of target heights. In this section equations for transferring data acquired at $(r, s)$ with a squint $\psi$ to zero-Doppler $\left(r_{0}, s_{0}\right)$ image coordinates are derived, and the sensitivity to look direction variations discussed.

The sensor path is approximated by (5). A coordinate system with $\hat{x}=\hat{t}_{0}, \hat{y}=\hat{b}_{0}$, and $\hat{z}=-\hat{n}_{0}$ is used, see Fig. 2. The origin of the $s$-coordinate is, contrary to (3), chosen so that $s=0$ corresponds to observing the target at the squint-angle $\psi$. Assuming a right-looking system the sensor to target vector can be written as

$$
\bar{r}=\left(\begin{array}{c}
r_{x} \\
r_{y} \\
r_{z}
\end{array}\right)=r\left(\begin{array}{c}
\sin \psi \\
-\cos \psi \sin \theta \\
-\cos \psi \cos \theta
\end{array}\right)
$$

where $\theta$ is the look-angle relative to the osculation plane of the orbit.

Closest approach - known target height

The length of the projection of the center-of-curvature to target vector onto the $(x, z)$-plane is

$$
R_{x z}=\sqrt{\left(R_{\kappa}+r_{z}\right)^{2}+r_{x}^{2}}
$$

By inspecting Fig. 2 the along-track coordinate, $s_{0}$, corresponding to closest approach is found to be

$$
s_{0}=R_{\kappa} \arctan \frac{r_{x}}{R_{\kappa}+r_{z}}
$$

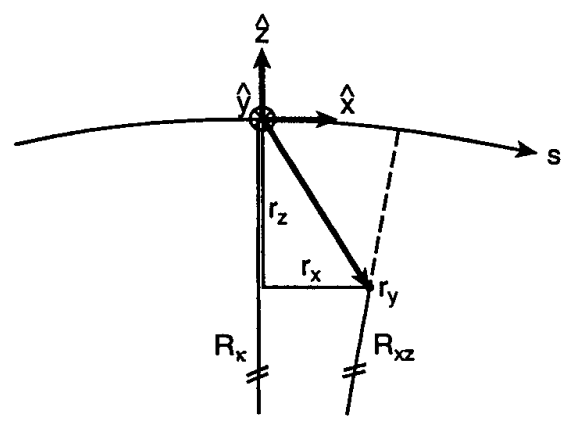

Fig. 2: Satellite track with a radius of curvature, $R_{\kappa}$, approximated by a circle in the osculation plane $(x, z)$, where $\hat{x}=\hat{t}_{0}, \hat{y}=\hat{b}_{0}$, and $\hat{z}=-\hat{n}_{0}$. A target is assumed at a known position $\left(r_{x}, r_{y}, r_{z}\right)$.

where $r_{z}<0$. Again, using Fig. 2, the closest approach distance, $r_{0}$, is found to be

$$
r_{0}=\sqrt{\left(R_{\kappa}-R_{x z}\right)^{2}+r_{y}^{2}}
$$

Thus, in transferring $(r, s, \psi)$ to a zero-Doppler geometry $r_{0}$ is given by (9), and $s$ is shifted by $s_{0}$ given by (8).

\section{Closest approach - unknown target height}

The partial derivatives of the sensor target vector $\bar{r}$ with respect to $\theta$ is

$$
\frac{\partial \bar{r}}{\partial \theta}=\left(\begin{array}{c}
0 \\
-r \cos \psi \cos \theta \\
r \cos \psi \sin \theta
\end{array}\right)=\left(\begin{array}{c}
0 \\
r_{z} \\
-r_{y}
\end{array}\right)
$$

Using (8), (10), and (6) it is seen that

$$
\begin{aligned}
\frac{\partial s_{0}}{\partial \theta} & =\frac{\partial s_{0}}{\partial r_{z}} \frac{\partial r_{z}}{\partial \theta}=\frac{R_{\kappa} r_{y}}{R_{x z}^{2}} r_{x} \\
& =-\frac{R_{\kappa} r^{2} \sin \theta}{2 R_{x z}^{2}} \sin 2 \psi
\end{aligned}
$$

This shows that to first order, the along track error caused by not knowing the target height is a linear function in $\psi$. Using (9), (7), and (10) the partial derivatives of the closest approach distance with respect to $\theta$ are found

$$
\begin{aligned}
\frac{\partial r_{0}}{\partial \theta} & =\frac{\partial r_{0}}{\partial r_{y}} \frac{\partial r_{y}}{\partial \theta}+\frac{\partial r_{0}}{\partial R_{x z}}\left(\frac{\partial R_{x z}}{\partial r_{x}} \frac{\partial r_{x}}{\partial \theta}+\frac{\partial R_{x z}}{\partial r_{z}} \frac{\partial r_{z}}{\partial \theta}\right) \\
& =-R_{\kappa} \frac{r_{y}}{r_{0}}\left(1-\frac{R_{\kappa}+r_{z}}{R_{x z}}\right) \\
& \approx \sin \theta_{0} \frac{s_{0}^{2}}{2 R_{\kappa}}
\end{aligned}
$$

where $\theta_{0}$ is the look angle at closest approach. It is seen that to first order, the slant range error caused by not knowing the target height is a quadratic function in $\psi$. 
Table 1: Image shifts $s_{0}$ (along-track) and $r-r_{0}$ (slant-range) for a transformation from a squint angle $\psi$ to a zero-Doppler geometry. An ERS geometry with $H=790 \mathrm{~km}, r=850 \mathrm{~km}$, near equator is used, $\left(\kappa_{h}=0\right)$. The transformation errors, $\Delta_{s}$ and $\Delta_{r}$, are for a target offset $2000 \mathrm{~m}$ from the reference sphere, $R_{e}=6370 \mathrm{~km}$.

\begin{tabular}{|c|c|c|c|c|}
\hline \multicolumn{5}{|c|}{ Impact of a $2000 \mathrm{~m}$ target height } \\
\hline $\begin{array}{c}\psi \\
{[\mathrm{deg}]}\end{array}$ & $\begin{array}{c}s_{0} \\
{[\mathrm{~m}]}\end{array}$ & $\begin{array}{c}r-r_{0} \\
{[\mathrm{~m}]}\end{array}$ & $\begin{array}{c}\Delta_{s} \\
{[\mathrm{~m}]}\end{array}$ & $\begin{array}{l}\Delta_{r} \\
{[\mathrm{~m}]}\end{array}$ \\
\hline $0.1^{\circ}$ & 1669 & 1.5 & -0.46 & 0.0004 \\
\hline $3.0^{\circ}$ & 50055 & 1311.9 & -13.89 & 0.3633 \\
\hline
\end{tabular}

To describe the impact of not knowing the target elevation, the along-track error, $\Delta_{s}$, and the slant-range error, $\Delta_{r}$, are calculated for a $2000 \mathrm{~m}$ elevation error when going from the squint angles $\psi=0.1^{\circ}$ and $\psi=3.0^{\circ}$ to a zero-Doppler geometry, see Table 1 . For a C-band SAR at a $790 \mathrm{~km}$ altitude the squint angles corresponds to Doppler frequencies of $460 \mathrm{~Hz}$ (yaw-steered ERS example) and $13880 \mathrm{~Hz}$ (non yaw-steered satellite, e.g. Radarsat or JERS-1). The target offset of $2000 \mathrm{~m}$ from the reference sphere corresponds to $\Delta \theta \approx 0.35^{\circ}$.

It is seen that for ERS, it is not critical to know the target height. For Radarsat, though, the effect is pronounced. Also note, that the difference between an ellipsoidal Earth model and a local spherical approximation is much smaller than typical height variations in the terrain.

\section{EARTH ROTATION - HORIZONTAL CURVATURE}

During focusing both the vertical orbit curvature, $\kappa_{v}$, and the horizontal curvature, $\kappa_{h}$, should be accounted for. If not de-focusing will occur, but also transformation between squint angles cannot be done properly.

The orbit curvature, $R_{\kappa}$, and rotation, $\alpha$, of the osculation plane of the orbit is

$$
\begin{aligned}
R_{\kappa} & =\frac{1}{\sqrt{\kappa_{v}^{2}+\kappa_{h}^{2}}}=\frac{1}{\kappa_{v} \sqrt{1+c_{\kappa}^{2}}} \\
\alpha & =\tan c_{\kappa}
\end{aligned}
$$

where $c_{\kappa}=\kappa_{h} / \kappa_{v}$, see Fig. 1. The combined effect of using a wrong radius of curvature and a wrong orientation of the osculation plane are shown in Table 2 for a horizontal curvature typical for ERS near the poles, i.e. $c_{\kappa}=0.15$.

\section{Radius of curvature}

By calculating the first and second order partial derivatives of (8) with respect to $c_{\kappa}$, a weak quadratic dependence on $c_{\kappa}$ is found. To first order the dependence on $\psi$ is linear. For $\psi=0.1^{\circ}, c_{\kappa}= \pm 0.15$, and the parameters from Table 1 the
Table 2: Image shifts $s_{0}$ (along-track) and $r-r_{0}$ (slant-range) for a transformation from a squint angle $\psi$ to a zero-Doppler geometry for ERS data near the poles, $\left(c_{\kappa}=0.15\right)$. The transformation errors, $\Delta_{s}$ and $\Delta_{r}$, arise if the horizontal curvature is ignored. Otherwise, the geometry and radar parameters equals those used for Table 1.

\begin{tabular}{r|rrrc}
\hline \hline \multicolumn{4}{c}{ Horizontal orbit curvature $\left(c_{\kappa}=0.15\right)$} \\
\hline $\begin{array}{c}s_{0} \\
{[\mathrm{deg}]}\end{array}$ & $\begin{array}{c}r-r_{0} \\
{[\mathrm{~m}]}\end{array}$ & \multicolumn{1}{c}{$\Delta_{s}$} & \multicolumn{1}{c}{$\Delta_{r}$} \\
{$[\mathrm{~m}]$} & \multicolumn{1}{c}{$[\mathrm{m}]$} & {$[\mathrm{m}]$} \\
\hline $0.1^{\circ}$ & 1681 & 1.5 & 11.8 & -0.010 \\
$3.0^{\circ}$ & 50406 & 1320.1 & 350.5 & -9.187 \\
\hline
\end{tabular}

along track mis-location is found to be $2.4 \mathrm{~m}$

Orientation of osculation plane

Ignoring the $\pm 9^{\circ}$ variations of the orientation of the osculation plane of the orbit due to the horizontal orbit curvature corresponds to up to a factor 30 larger $\theta$ variations than unknown terrain. This causes $\pm 10 \mathrm{~m}$ along track mis-locations even for a small squint of $0.1^{\circ}$ as for ERS. This is of the same order of magnitude as the combined effect of all other along track error sources, [1].

\section{CONCLUSIONS}

Equations for transformation of data acquired with squint to a zero-Doppler geometry are derived. For satellites, the transformation depends on the squint angle and the look angle between the osculation plane of the orbit and the target. An unknown target height is not a concern for the yaw-steered ERS, but should be considered for non yaw-steered systems if the highest geometrical accuracy is required. In both cases, Earth rotation has to be taken into account in the along-track transformation of data to a zero-Doppler geometry, in order to keep the error well below $10 \mathrm{~m}$.

\section{REFERENCES}

[1] J.J. Mohr and S.N. Madsen, "Automatic generation of large scale ERS DEMs and displacement maps," in http://www.esrin.esa.it/fringe99, Liege, Belgium, 1999.

[2] J.C. Curlander, "Location of spaceborne SAR imagery," IEEE Trans. on Geoscience and Remote Sensing, vol. 20, no. 3, pp. 359-364, July 1982.

[3] S.N. Madsen and H. Zebker, "Imaging radar interferometry," in Manual of Remote Sensing, Floyd. M. Henderson and Anthony J. Lewis, Eds., vol. 2, chapter 6, pp. 359-380. John Wiley \& Sons, Inc., 3rd edition, 1998.

[4] G.A. Korn and T.M. Korn, Mathematical Handbook for Scientist and Engineers, McGraw-Hill Book Company, Inc., 1961. 